\title{
TIDAL EFFECTS IN CLOSE BIMARY STARS
}

\author{
Jean-Paul ZAHN \\ Observatolres du Pic du Midi et de Toulouse
}

\section{INTRODUCTION}

This review addresses the question of what can be learned about the stellar interiors from the study of the tidal effects in close binary stars.

The basic mechanism of tidal interaction is sufficiently well known that we need only here to recall the main results. For more detail, we refer the reader to the classical monography written by Kopal (1959).

In a binary star, each component is submitted to a tidal potential $U$, which can be expanded in spherical functions for its spatial dependence, and in Fourier series for its temporal behavior. such expansion takes the form

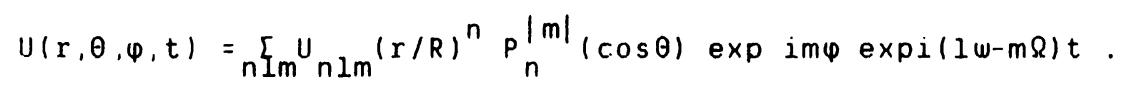

For convenience, the axis of the polar coordinates $r, \theta, \varphi$ will be chosen perpendicular to the orbital plane. $R$ is the mean radius of the star, $w$ the mean orbital angular velocity and the integers $n, l, m$ take all values satisfying the inequalities $n \geqslant 2,|m| \leqslant n$.

The celestial mechanics provides expressions for the amplitudes Unlm of this expansion in terms of the mass of the companion star and the parameters characterizing the orbit.

Since the star is distorted by that tidal potential, its outer gravitational field contains a non-spherical component, whose potential can likewise be expanded as:

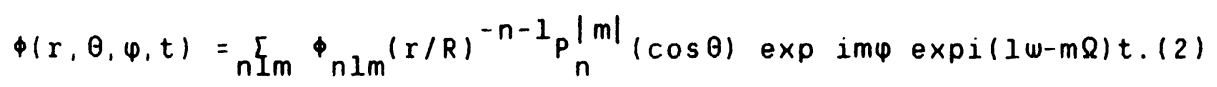

The amplitudes $\phi_{n l m}$ of this perturbing potential are proportional

A. Maeder and A. Renzini (eds.), Observational Tests of the Stellar Evolution Theory, 379-389.

(C) 1984 by the IAU. 
to the amplitudes $U$, of the tidal potential, and the proportionality coefficientes which link them reflect the internal structure of the star, and more generally its physical properties. Since the companion star moves in that perturbing potential , it can be used as a test particle to explore this field, and hence to determine the coefficients mentioned above. This provides one of the most powerful means of probing the interior of the stars.

Before we proceed, let us mention that the rotational distorsion can be treated in the same way, at least as long as the centrifugal force derives from a potential; that potential then plays the same role as the tidal potential, and it also induces a perturbing field.

\section{APSIDAL MOTION}

To first approximation, ignoring the dynamical effects and neglecting all dissipation mechanisms, one has

$$
\phi_{n l m} / U_{n l m}=2 k_{n}
$$

where the $k$ are constants which depend only on the mass distribution inside the stâr (see Kopal 1959).

By far the largest contribution to the perturbing potential comes from the quadrupolar moment of this mass distribution $(n=2) ; i t$ creates a force in $r^{-4}$ which causes a slow uniform rotation of the semi-major axis in an eccentric orbit. For this reason, $k_{2}$ is called the apsidal motion constant. Its value would be 0.75 for a fomogeneous star; in real stars, $k_{2}$ drops below $10^{-2}$.

Both stars contribute of course to this apsidal motion, whose period $P$ aps is related to the apsidal motion constants $\left(k_{2}\right){ }_{1}$ and $\left(k_{2}\right)_{2}$ of the two components by

$$
P_{\text {aps }}=c_{1}\left(k_{2}\right)_{1}+c_{2}\left(k_{2}\right)_{2}
$$

to that degree of approximation $(n=2)$. The coefficients $c_{i}$ are given by :

$$
\begin{gathered}
c_{i}=\left(R_{i} / a\right)^{5}\left[15\left(M_{3-i} / M_{i}\right)\left(1+\frac{3}{2} e^{2}+\frac{1}{8} e^{4}\right)\left(1-e^{2}\right)^{-5}\right. \\
\left.+\left(\Omega_{i} / w\right)^{2}\left(1+M_{3-i} / M_{i}\right)\left(1-e^{2}\right)^{-2}\right] .
\end{gathered}
$$

The first term of this expression is due to the tidal potential. and the second to the rotational distorsion; $M_{i}$ and $R_{j}$ are the masses and radii of the stars, $\Omega$ their angular velocities lsupposed uniform here, with the rotation axis being perpendicular to the orbital plane), $P$ is the period of the orbital motion $(P=2 \pi / w)$, a thesemimajor axis and e the eccentricity. 
There is also a relativistic contribution to the apsidal motion, but it is in general negligible; one exception is V889 Aql, a star studied by Gimenez and Scaltriti (1982) (and of course the Sun when considering the orbit of Mercuryl.

The severe $a^{5}$ dependence of $P$ explains why the apsidal motion can only be detected in very clöges binaries. For reasons that will become clear later on, it is difficult to observe in binaries which contain lower main sequence stars.

For a given binary showing this apsidal motion, the coefficients $c_{\text {c }}$ can in principle be determined from the observations. The ideal càse is that of an eclipsing binary, for which the fractional radii $R_{i} / a$ can be measured directly. If the rotational velocities $\Omega$ cannot be derived from the width of the spectral lines, some assumptions must be made (corotation with the orbital motion, for instance), but they do not have a crucial influence on the result, at least for not too extreme mass ratios.

Once the coefficients $c$ have been calculated, Eq. 4 provides a weighted mean of the apsidal motion contants, which can be compared with the theoretical predictions. This procedure is known as the apsidal motion test. The first of such comparisons were made by the pioneers in this field: Cowling (1938), Sterne (1939), Russel (1939), Luyten el al. (1939), and Kopal (1940); they found a reasonable agreement with the stellar structure theory, which was then in infancy, although they were somewhat disquietened because Eddington's standard model could not account for all the stars.

Shortly after the publication by Kushwaha (1957) of much improved stellar models for upper main sequence stars, a new comparison was carried out by schwarzschild (1958). He found a trend, for the then observed stars, to have a smaller $k$, than that predicted by the models ; the discrepancy, as measured by $\Delta \log k_{2}=\left(\log k_{2}\right)$ theory - (log $k_{2}$ ) observation, was of order 0.5 , the reference models being those of zero age main sequence (ZAMS) stars. But since $k_{2}$ decreases as the star evolves off the main sequence, the star becoming more centrally condensed, Schwarzschild concluded that the observed discrepancy could be ascribed to evolutionary effects.

This interpretation was later questioned by Kopal (1965), and new comparisons were made by Petty (1973), Stothers (1974), 0de11 (1974) and Monet (1980), who used more refined models of various chemical compositions, and tried different opacities.

The most recent investigation is due to Gimenez and Garcia-Pelayo (1982) ; they rediscuss the available data, retain 55 eclipsing binaries, determine their apsidal motion period by a more refined method and compare the results with current ZAMS models. They find a very strong correlation between $\Delta \log k_{2}$ and $\Delta \log g$, the latter 
quantity refering to the gravity : $\Delta \log g=(\log g)$ ZAMS - (log $g)$ present state. Moreover, the regression line, which has a slope of nearly +1 , passes through the origin $\left(\Delta \log k_{2}, \Delta \log g\right)=(0.0)$.

There is therefore little doubt left that the departures of the observed apsidal motion constants from those predicted by ZAMS models is due primarily to the evolution of the stars. However, the standard evolutionary sequences seem unable to explain the occurrence of rather large values of $\Delta \log k_{2}$, up to 0.8 .

Two explanations are presently offered to account for these large deviations. One, already advocated by stothers (1974), and again supported by Jeffery at this meeting, is that the cox-stewart opacities are inadequate and ought to be replaced by the carson opacities, which seem to lead to a better agreement for the evolved stars. Another interesting suggestion was made by odell (1974), namely that some mixing occurs near the convective core of the stars; evolutionary calculations performed in allowing such mixing also yield smaller values of $\mathrm{K}_{2}$.

At the present stade, the question can hardly be considered as settled, and one should therefore encourage both further observations and further theoretical work.

\section{CIRCULARISATION OF THE ORBITS AND SYNCHRONISATION OF THE ROTATION}

\subsection{Stars with convective envelopes}

Proceeding further with the analysis of the tidal interactions in a binary star, the next step is to consider the effets which arise from viscous friction or radiative damping. Let us do this first while still ignoring the contribution of the inertial terms $;$ in other words, we shall deal only, as before, with the equilibrium tide.

The dissipation mechanisms act to delay the tide by an angle $\alpha$ which is called the tidal lag; its expression has been established by Darwin (1879) for a viscous stars:

$$
\alpha=\frac{Q-w^{3}}{t_{F}} \quad \frac{R}{G M} .
$$

The tidal lag is inversely proportional to the friction time $t_{F}$, which is given by :

$$
t_{F}=R^{2} / v
$$

$\checkmark$ being the viscosity, and neglecting for the present purpose factors of order unity. 
The same expression (6) can by used to predict the tidal lag due to radiative damping, the corresponding friction time then being the Kelvin-Helmholtz time $t_{F}=R / k \sim G M / R L$ (k : mean radiative conductivity, $L$ : luminosity of the starl.

It is that tidal lag which is responsible for the exchange of angular momentum between the rotation of the stars and their orbital motion. This has two observable consequences : the decrease of the eccentricity of the orbit, and the synchronization of the rotation of the stars win their orbital revolution. The time scales for these two phenomena are given by:

$$
t_{\text {circ }}^{-1}=-d \operatorname{lne} / d t=21 / 2 \quad k_{2} / t_{F} \quad q(1+q) \quad(R / a)^{8}
$$

for the circularization, and

$$
t_{\text {sync }}^{-1}=-d \ln (Q-w) / d t=6 k_{2} / t_{F} \quad q^{2} M R^{2} / I \quad(R / a)^{6}
$$

for the synchronization. Here $q$ is the mass ratio of the considered star with respect to its companion, and $I$ is its momentum of inertia. I In Eq. 8 the contribution of the second star has been omitted for simplicity sake.)

When applying these expressions to the dissipation mechanisms mentioned above, namely viscous friction and radiative damping, one obtains time scales which are much too long to explain the observations. The only mechanism which, for the equilibrium tide, yields time scales that are compatible with the observations, turns out to be the turbulent friction due to thermal convection Izahn 1966). If the considered star has a çonvegtive envelope of sufficient depth, the friction time is $t_{f} \backsim\left(M R^{2} / L\right)$, within the uncertainties of the stellar convection theory, and this time is extremely short (about 1 year for main sequence stars).

It is because the turbulent stresses are so efficient that most close binaries with convective envelopes have circular orbits. (For this reason, consequently, very few of such binaries can be submitted to the apsidal motion test.l The qualitative difference in behavior between upper and lower main sequence binaries is striking, and may even be used to determine the mass above which those stars no longer prossess an outer convection zone (Zahn 1966). But it is only recently that the quantitative agreement between theory and observations for late type main sequence binaries has been checked by Koch and Hrivnak (1980) : they performed computer simulations of the dynamical evolution of a sample of hypothetical binary systems, and found that their circularization proceeds on a time scale which is compatible with the observations. 
Another proof of the theory is offered by the BY Dra stars. The rotation period of these $K$ and $M$ dwarfs can be determined accurately through their photometric variations, which are attributed to spots on the rotating surface. Bopp and Fekel (1977) have shown that the incidence of binaries among those stars is rather high, and that most BY Dra binaries are synchronized with their orbital motion. The two of them which depart significantly from synchronism lincluding BY Dra itself) may be interpreted as systems which are young (they also have eccentric orbits). as was discussed by Bopp at al. (1980) and by Edwards (1983).

One may thus consider as well established that the physical mechanism which is responsible for the orbital circularization in lower main sequence binaries is the turbulent friction in the convective envelope of those stars, acting on the equilibrium tide.

\subsection{Stars with radiative envelopes}

For the upper main sequence binaries, another mechanism must be invoked, namely radiative damping on the dynamical tide (Zahn 1975). It is well known that a star, which is a self-gravitating compressible body, can oscillate in a variety of eigenmodes, which have been classified by Cowling in p-modes (or acoustic modes) and g-modes (or gravity modes). The latter have the largest period, and they may therefore enter in resonance with the periodic tidal potential, if the considered star is a binary component. In practice, the amplitude of such resonances is severely limited by radiative damping, in the outer part of the envelope where the radiative relaxation time becomes rather short.

Since the modes which are involved in those resonances are of high order, an asymptotic theory can be applied to study them and to predict their amplitude. In the limit of a very long tidal period (i.e. close enough to synchronism), this amplitude does not depend on the details of the damping mechanism involved, but only on a series of structural parameters $E_{n}$ which are much like the apsidal motion constants $k_{n}$.

For the dynamical tide, the amplitude ratio between the fourier components of the perturbing potential and those of the tidal potential then takes the limiting form :

$$
{ }_{n l m}^{\prime} U_{n l m}=\left(3^{-1 / 2}+i\right) E_{n}\left[(1 w-m Q)\left(R^{3} / G M\right)^{1 / 2}\right]^{8 / 3}
$$

It is the imaginary part of this expression which is responsible for the exchange of angular momentum; the real part contributes, although negligibly, to the apsidal motion. The coefficients $E_{p}$ are very sensitive to the structure of the star, and in particular to the size of its convective core. For zero age maip sequence stars, its value ranges from $2.410^{-9}$ (for $1.6 \mathrm{M}_{\theta}$ ) to $3.510^{-6}$ (for $15 \mathrm{M}_{\theta}$ ). 
We refer the reader to the original paper (Zahn 1977) for the expressions giving the relevant synchronization and circularization time scales. Here we shall repeat only the parameters which may be the most useful for the comparison with the observations. Table 1 lists the period $P$ and the separation a below which a binary will be respectively šnchronized and circular̆ized during its main sequence life span. (The table gives the directly measurable fractional radius $\mathrm{R} / \mathrm{a}$ rather then $\mathrm{a}$; the binaries are assumed to consist of two ideñtical components. $\mathcal{Y}$

Several papers have been devoted recently to the verification of the predictions based on this theory, using the observational data available for detached binaries. I In semi-detached and contact binaries, the mass transfer is expected to be much more effective than the tidesl.

\section{Iable 1}

critical separations and oeriods for the sunchronization and circularization in close binaries

Synchronization

$\begin{array}{ccc}\text { Mass }\left(M_{0}\right) & R / a c & P_{C} \text { (days } \\ 1.0 & 0.022 & 30 \\ 1.6 & 0.164 & 1.21 \\ 2 & 0.142 & 1.59 \\ 3 & 0.147 & 1.92 \\ 5 & 0.153 & 2.19 \\ 10 & 0.150 & 3.30 \\ 15 & 0.142 & 3.98\end{array}$

Circularization

Cause

Causes : (1) Turbulent friction acting on the equilibrium tide in the convective envelope ; (2) Radiative damping acting on the dynamical tide in the radiative envelope.

For the circularization, the observations show that most systems with $R / a \geqslant 0.25$ have circular orbits, as shown by Giuricin et al. (1984b). This limit is slightly higher than that predicted by the theory outlined above $(0.200$ to 0.225 depending on the mass), suggesting that the tidal coefficients $E$ are probably higher than those inferred from the zero age main sequence models. A noticeable exception is $\alpha$ Vir, for which $e=0.14$ whereas $R / a=0.29$; one is therefore tempted to interpret this system as a young unevolved binary. 
For the degree of synchronization, the situation is more complex. one observes a marked tendency for synchronism above $R / a=0.15$, in agreement with the theory; however, as was noticed by Rajamohan and Venkatakrishnan (1981) and by Giuricin et al. (1984a), below that limit and down to $R / a=0.05$, both synchronized and non synchronized systems exist. Rajamohan and Venkatakrishnan invoke high subsurface magnetic fields to account for this stronger than expected synchronization. A more plausible explanation is that the surface layers, which experience a higher torque per unit mass, are synchronized much faster than the interior of the star. If this is confirmed, the observations can yield an upper limit of the differential rotation which is tolerated in a star.

Gimenez and Andersen (1983), and also Giuricin et al. (1984a), observe that in eccentric binaries synchronism is achieved for a velocity which is larger than the Keplerian velocity $v_{k}=w a$. Such a deviation is expected for any type of tidal friction; its exact value, once determined, can also be used to identify the physical mechanism which is responsible for it. However, as we have seen, there is little doubt left that this mechanism is indeed the radiative damping acting on the dynamical tide.

\section{CONCLUSION}

The study of the tidal interactions in close binaries has already provided one of the most valuable checks of the stellar structure theory. More will certainly be learned, through better determinations of an increasing number of apsidal motion constants; for instance they may put observational constraints on the mild mixing processes which are now suspected in radiative interiors, due to convective overshooting or hydrodynamical instabilities.

And a new field of research has opened with the observation of the degree of circularization and synchronization, which contains potentially a precious information on the age and the evolutionary state of a given system, even though some assumptions have to be made on the yet unknown initial conditions. To exploit this information completely, a new approach is probably needed, involving the simulation of the whole dynamical history of the considered binary. And the theory itself must be refined, for example to take explicitely the rotation into account, for it induces a variety of oscillation modes which have not be considered so far, namely the quasi-toroidal modes (see Provost et al. 1979).

\footnotetext{
May I conclude by expressing the hope that many more colleagues, both observers and theoreticians, will be seduced by this exciting subject !
} 


\section{REFERENCES}

Bopp,B.W.,Fekel,F.:1977, Astron. J. 82, 490.

Bopp,B.W. Noah,P.V.,Klimke, A.:1980, Astron. 3. 85, 1386 .

Cowling, T.G.:1938, Monthly Notices Roy. Astro. Soc. 98, 734 .

Darwin,G.H.:1879, Phil. Trans. Roy. Soc. 170, 1.

Edwards, D.A.:1983, Astron. Astrophys. 123, 316.

Gimenez,A., Scaltriti,F.:1982, Astron. Astrophys. 115, 321.

Gimenez,A.,Garcia-Pelayo,J.M.:1982, Binary and Multiple Stars as Tracers of Stellar Evolution, 37 ( $Z$. Kopal and J. Rahe eds.) $D$. Reidel Publ. Co, Holland.

Gimenez,A.,Andersen,J.:1983, Réunion de Strasbourg sur "Les Etoiles Binaires dans le Diagramme $H R^{*}$.

Giuricin,G.,Mardirossian,F. Mezzetti,M.:1984a, Astron. Astrophys. (in printl.

Giuricin,G.,Mardirossian,F.,Mezzetti,M.:1984b, Astron. Astrophys. (in print).

Koch,R.M.,Hrivnak,B.J.:1981, Astron. J. 86, 438.

Kopal;z.:1940, Harvard Circ. $N^{*} 441$.

Kopal,Z.:1959, Close Binary Systems, Chapman Hall, London.

Kopal,2.:1965, Adv. Astron. Astrophys. 3, 89.

Kushwaha,R.S.:1957, Astrophys. J. 125, 242.

Luyten,W.J., Struve, I. Morgan,W.W.: 1939, Publ. Yerkes Obs. 7, 251.

Monet,D.G.:1980, Astrophys. J. 237, 513.

Odell,A.P.:1974, Astrophys. J. 192, 417.

Petty,A.F.:1973, Astrophys. Space Sci. 211, 189.

Provost, J.,Berthomieu, G.,Rocca,A.:1981, Astron. Astrophys. 94, 126.

Rajamohan, R, Venkatakrishnan,P.:1981, Bull. Astron. Soc. India 9, 309 .

Russel,H.N.:1939, Astrophys. J. 90, 641. 
Sohwarzschild,M.:1958, Structure and Evolution of the Stars, Princeton Univ. Press.

Shu,F.H.,Lubow, S.H.:1981, Ann. Rev. Astron. Astrophys. 19, 277.

Sterne,T.E.:1939, Monthly Notices Roy. Astron. Soc. 99, 662.

Stothers, R.:1974, Astrophys. J. 194, 651.

Zahn, J.-P.:1966, Ann. Astrophys. 29, 313, 489 and 565.

Zahn, J.-P.:1975, Astron. Astrophys. 41, 329.

Zahn, J.-P.:1977, Astron. Astrophys. 57, 394.

\section{DISCUSSION}

Meste1: I believe there is support for tidal synchronization from observations (especially by Abt et al) on A-stars in binary systems. Wide binaries have normal, rapidly rotating A-stars. Intermediate systems have A-stars with Am spectra, for which slow rotation is believed to be essential; spin-orbit synchronization will indeed produce slow rotators. In close binaries, synchronization produces rapid rotators, so that the A stars should again be normal.

Spruit: Did you say that in early-type binaries there is agreement with predictions as far as circularization is concerned?

Zahn: Yes.

Andersen: Two questions from the observer's point of view:

1) What we can observe spectroscopically is of course the rotation of the surface layers (and we can say only whether it agrees with synchronism to within $\pm 10 \%$ or so). If I understood correctly, you suggest that the surface layers may well be synchronized while the interior is still rotating more rapidly, while my recollection of a similar recent review by Savonije (Cambridge, Aug. 1983) is the reverse?

2 ) Would you always expect $t$ < $<$ for stars of the same type (cf. our poster on TZ For, where $\mathrm{M}_{1} \approx \mathrm{F}_{2}$, both stars are convective, orbit is circular, and the larger star is synchronized while the smaller one is not)?

Zahn: 1) The torque per unit mass is larger near the surface than in the interior. One expects therefore the surface layers to be synchronized faster, if they decouple from the interior.

2) Yes, $t_{\text {sync }}<t_{\text {circ }}$ as long as the mass ratio is not too extreme. 
Mayor: You mention a limiting period of $\mathrm{P}=5.6$ days for orbital circularization of a system with one solar mass components. After how much time this circularization is achieved?

Zahn: The decay of the eccentricity is exponential in time. In a mainsequence 1 ife span (about $10^{10}$ years) the eccentricity will be reduced by a factor of $\mathrm{e}^{4} \sim 60$ for such a system. 